\title{
Disponibilidade, composição bromatológica e consumo de matéria seca em pastagem consorciada de Brachiaria decumbens com Stylosanthes guianensis
}

\author{
Luiz Januário Magalhães Aroeira(1), Domingos Sávio Campos Paciullo(1), Fernando César Ferraz Lopes ${ }^{(1)}$, \\ Mirton José Frota Morenz ${ }^{(1)}$, Eloísa Simões Saliba ${ }^{(2)}$, Janaína Januário da Silva( ${ }^{(2)}$ e Carlos Ducatti( ${ }^{(3)}$
}

(1)Embrapa Gado de Leite, Rua Eugênio do Nascimento, no 610, Dom Bosco, CEP 36038-330 Juiz de Fora, MG. E-mail: laroeira@cnpgl.embrapa.br, dscp@terra.com.br, fernando@cnpgl.embrapa.br, mirtonmorenz@yahoo.com.br (2)Universidade Federal de Minas Gerais, Av. Antônio Carlos, no 6.627, Campus Pampulha, CEP 31270-901 Belo Horizonte, MG. E-mail: saliba@vet.ufmg.br, janajanu@yahoo.com (3)Universidade Estadual Paulista "Júlio de Mesquita Filho", Fac. de Medicina Veterinária e Zootecnia, Caixa Postal 560, CEP 18618-000 Botucatu, SP. E-mail: ducatti@ibb.unesp.br

\begin{abstract}
Resumo - O objetivo deste trabalho foi avaliar a disponibilidade de forragem, a composição bromatológica, o consumo de matéria seca e a proporção de gramínea e leguminosa na dieta de vacas mestiças Holandês x Zebu, em pastagem consorciada de Brachiaria decumbens cv. Basilisk, Stylosanthes guianensis var. vulgaris cv. Mineirão e leguminosas arbóreas. Para estimativa da produção fecal, foram usados $10 \mathrm{~g} \mathrm{vaca}^{-1} \mathrm{dia}^{-1} \mathrm{de}$ óxido crômico, durante dez dias. Amostras de extrusa foram usadas para determinação da composição bromatológica e digestibilidade in vitro da matéria seca. A disponibilidade de matéria seca de forragem de $B$. decumbens variou com as condições climáticas, enquanto a de $S$. guianensis decresceu linearmente ao longo do período experimental. O consumo de matéria seca foi maior em maio de 2001 (1,9\% do peso do animal vivo) e não diferiu entre os demais meses (1,5\% do peso do animal vivo). Os baixos índices de consumo de matéria seca refletiram altos teores de fibra em detergente neutro (70,2\% a 79,4\%) e baixos coeficientes de digestibilidade in vitro de matéria seca $(42,1 \%$ a $48,0 \%)$ da forragem. O consumo de leguminosa variou entre $8,7 \%$ e $24,1 \%$ do total ingerido. $\mathrm{O}$ consumo de matéria seca esteve diretamente relacionado à porcentagem de leguminosa na pastagem, o que evidencia o potencial de uso de pastagens consorciadas para vacas leiteiras.
\end{abstract}

Termos para indexação: Brachiaria decumbens, Stylosanthes guianensis, ingestão de forragem, leguminosa forrageira, valor nutritivo.

\section{Herbage availability, chemical composition and dry matter intake in mixed pasture of Brachiaria decumbens with Stylosanthes guianensis}

\begin{abstract}
The objective of this work was to evaluate the herbage availability, nutritive value, dry matter intake and grass and legume percentage in diet of crossbred Holstein-Zebu cows, in pasture with Brachiaria decumbens cv. Basilisk, Stylosanthes guianensis var. vulgaris cv. Mineirão and tree legumes. To estimate the fecal output, it was used $10 \mathrm{~g} \mathrm{cow}^{-1}$ day $^{-1}$ of chromium oxide during ten consecutive days. Extrusa samples were used to determine the chemical composition and in vitro dry matter digestibility. B. decumbens availability varied with climatic conditions, while $S$. guianensis availability decreased linearly along the experimental period. Dry matter intake was higher in May/2001 (1.9\% body weight) and did not differ among other months (1.5\% body weight). Low dry matter intake values were related to low in vitro dry matter digestibility coefficients ( $42.1 \%$ to $48.0 \%$ ) and high neutral detergent fiber content (70.2\% to 79.4\%). Dry matter intake was directly related to legume percentage in the pasture. This observation could indicate the potential of mixed pasture for improving nutritive value in dairy cattle diet.
\end{abstract}

Index terms: Brachiaria decumbens, Stylosanthes guianensis, forage intake, forage legume, nutritive value.

\section{Introdução}

A pecuária brasileira, especialmente no Brasil central, tem como base o sistema de produção extensivo, com uso de pastagens exclusivas de gramíneas. Estimativas admitem que $80 \%$ a $90 \%$ das áreas de pasta- gens no País são constituídas por capins do gênero Brachiaria, e que a B. decumbens ocupa mais de $50 \%$ do total formado por essas gramíneas (Boddey et al., 2004). Grande parte das pastagens de braquiária cultivadas em diferentes regiões do Brasil, tem mostrado sinais de degradação em poucos anos de uso. A falta de 
persistência dessas gramíneas pode ser atribuída à forma extrativista de sua exploração, bem como a problemas de adaptação, ao manejo inadequado das diferentes espécies, à redução da fertilidade do solo, à susceptibilidade a pragas e à falta de adubação de reposição (Rodrigues, 1996).

As pastagens consorciadas de gramíneas e leguminosas forrageiras constituem boa opção, de baixo custo, para atenuar o problema da degradação das pastagens. O uso de leguminosas forrageiras com capacidade de fixação de $\mathrm{N}$ atmosférico por meio da simbiose com Rhizobium melhora a qualidade da liteira da pastagem, podendo fornecer grandes quantidades de $\mathrm{N}$ ao sistema solo-planta-animal (Giller \& Cadisch, 1995; Cantarutti et al., 2002). A contribuição pode ser feita pela transferência do $\mathrm{N}$ fixado para a gramínea, o que aumenta a capacidade de suporte da pastagem e prolonga sua capacidade produtiva (Cantarutti et al., 2002). Além disso, o $\mathrm{N}$ fixado pela leguminosa pode melhorar a qualidade da dieta (Costa, 1995) e aumentar a produção animal (Euclides, et al., 1998). Outra vantagem das leguminosas é a menor variação estacional no seu valor nutritivo, em comparação com as gramíneas forrageiras (Jingura et al., 2001).

O fracasso na adoção e utilização de pastagens consorciadas, em geral, é atribuído à baixa persistência das leguminosas nas pastagens, o que está associado à falta de técnicas de manejo específicas ou eficientes para essas pastagens, e à adubação inadequada. Embora o cultivo de leguminosas forrageiras seja considerado desgastado pelos insucessos observados no passado, sua adoção torna-se cada dia mais importante e factível, graças às novas práticas de cultura e de manejo, associadas a novas cultivares geradas pela pesquisa (Barcellos et al., 2003).

O principal fator nutricional que controla a produção é a quantidade de alimento oferecido em excesso, que o animal é capaz de ingerir diariamente. Em geral, o baixo consumo tem sido observado em animais mantidos em pastagens de Brachiaria spp., estando tal fato relacionado, geralmente, aos baixos teores de proteína da forragem, entre outros fatores (Lascano \& Euclides, 1996).

O uso de pastagens consorciadas de gramíneas e leguminosas pode aumentar a proteína da dieta, o consumo de matéria seca e o desempenho do animal. A literatura registra, no entanto, alguns resultados insatisfatórios para o consumo e ganho de peso de animais, em pastagens consorciadas. Esses resultados po- dem estar relacionados com a baixa palatabilidade da leguminosa, sua pequena participação na composição botânica do pasto e ao consumo variável de leguminosa ao longo das estações do ano (Pereira et al., 1992).

O objetivo deste trabalho foi avaliar a disponibilidade e o consumo de matéria seca, a composição bromatológica e a proporção de gramínea e leguminosa na dieta de vacas mestiças Holandês x Zebu, em pastagem consorciada de Brachiaria decumbens cv. Basilisk, Stylosanthes guianensis var. vulgaris cv. Mineirão e leguminosas arbóreas.

\section{Material e Métodos}

O experimento foi realizado na Embrapa Gado de Leite, em Coronel Pacheco, MG, durante o período de janeiro de 2001 a novembro de 2002. O sistema silvipastoril (SSP) foi estabelecido num Latossolo Vermelho-Amarelo distrófico, em área de topografia montanhosa, com declividade de aproximadamente $30 \mathrm{~cm} \mathrm{~m}^{-1}$. A precipitação média mensal é de $60 \mathrm{~mm}$ e temperatura média de $17^{\circ} \mathrm{C}$, de abril a setembro, e de $230 \mathrm{~mm}$ e $24^{\circ} \mathrm{C}$, de outubro a março. As leguminosas arbóreas Acacia mangium, Acacia angustissima, Mimosa artemisiana e o Eucaliptus grandis foram plantadas em faixas de $10 \mathrm{~m}$, intercaladas com faixas de $30 \mathrm{~m}$, plantadas com Brachiaria decumbens e Stylosanthes guianensis.

Antes do plantio, em novembro de 1997, aplicaramse $1.000 \mathrm{~kg} \mathrm{ha}^{-1}$ de calcário dolomítico, $600 \mathrm{~kg} \mathrm{ha}^{-1}$ de fosfato de Araxá, $25 \mathrm{~kg} \mathrm{ha}^{-1}$ de superfosfato simples, $100 \mathrm{~kg} \mathrm{ha}^{-1}$ de cloreto de potássio e $30 \mathrm{~kg} \mathrm{ha}^{-1}$ de FTE BR-16. As densidades de semeadura foram $10 \mathrm{e}$ $1 \mathrm{~kg} \mathrm{ha}^{-1}$ de $B$. decumbens e $S$. guianensis, respectivamente.

As avaliações de disponibilidade e consumo de MS foram realizadas em três piquetes com área de 0,5 ha.

Foi usado o delineamento experimental em blocos casualizados, com repetições constituídas por piquetes (três repetições) nas avaliações de disponibilidade, composição química e digestibilidade da forragem ou pelos animais, no caso das avaliações de consumo. Os tratamentos experimentais foram de janeiro de 2001 a novembro de 2002.

As avaliações de disponibilidade de matéria seca (MS) e de composição botânica foram realizadas durante os meses de janeiro, março, maio, outubro e dezembro de 2001 e janeiro, maio, julho e novembro de 2002 . As amostras foram obtidas com auxílio de uma moldura 
quadrada de 0,5 m de lado, lançada vinte vezes, ao acaso, em cada piquete. A forragem contida em cada moldura foi cortada a $5 \mathrm{~cm}$ do nível do solo e levada ao laboratório, onde foi separada, manualmente, em B. decumbens, S. guianensis e material morto. De cada espécie, foi retirada uma subamostra para separação das frações lâmina foliar e bainha foliar + colmo. Cada componente foi pesado e secado em estufa a $65^{\circ} \mathrm{C}$, até peso constante, para determinação da MS.

As extrusas para determinação da composição bromatológica e digestibilidade foram coletadas de uma vaca fistulada no esôfago, que permaneceu durante os dez dias de cada avaliação na área experimental. As coletas foram realizadas pela manhã, durante os últimos seis dias de cada período de pastejo, de modo a totalizar seis amostras durante cada período. As amostras foram analisadas quanto aos teores de proteína bruta (PB), de acordo com Queiroz \& Silva (2002); fibra em detergente neutro (FDN) e fibra em detergente ácido (FDA), segundo Soest et al. (1991); e digestibilidade in vitro da matéria seca (DIVMS), conforme com Tilley \& Terry (1963).

As estimativas de consumo foram realizadas durante os meses de janeiro, maio e dezembro de 2001 e janeiro, maio e novembro de 2002. Os piquetes foram manejados com vacas mestiças Holandês x Zebu, secas, durante dez dias em cada época. O intervalo de desfolha foi de 40 dias, período em que os piquetes permaneceram sem animais. $\mathrm{O}$ ajuste do número de animais, por piquete, durante os períodos de pastejo, foi feito de acordo com a disponibilidade de forragem estimada antes do início do período de avaliação, a fim de que ofertas de forragem com variação de $7 \%$ a $9 \%$ do peso vivo não limitassem o consumo de MS. Para isso, foram colocados dois animais piquete ${ }^{-1}$ durante a avaliação de maio de 2001, e de três animais piquete ${ }^{-1}$ durante os demais períodos avaliados.

As estimativas de consumo total de MS, para o cálculo da produção fecal, foram feitas com auxílio do óxido crômico. Foram fornecidas $10 \mathrm{~g}$ diárias de óxido crômico, em uma aplicação pela manhã, durante dez dias consecutivos. O consumo total de MS foi estimado a partir da fórmula:

Consumo de MS = produção fecal x 100/(100 - DIVMS).

Para conhecer as proporções de gramínea $\left(\mathrm{C}_{4}\right)$ e leguminosas $\left(\mathrm{C}_{3}\right)$ na dieta total dos animais, foi aplicada a técnica de absorção do carbono (Ludlow et al., 1976), que se baseia na diferença entre os ciclos fotossintéticos denominados $\mathrm{C}_{3}$ e $\mathrm{C}_{4}$. Nas estimativas de consumo, a análise da abundância isotópica de $\delta^{13} \mathrm{C}$, nas fezes, permite a identificação do carbono derivado das plantas $\mathrm{C}_{3}$ e $\mathrm{C}_{4}$.

Os dados obtidos foram submetidos à análise de variância, e as médias foram comparadas pelo teste de Tukey a 5\% de probabilidade.

\section{Resultados e Discussão}

Os rendimentos de MS de $B$. decumbens variaram com o período de avaliação, tendo sido maiores durante os meses de precipitações e temperaturas mais altas (janeiro a maio e novembro e dezembro), que decresceram, acentuadamente, durante os meses de outubro de 2001 e julho de 2002, período seco na Região Sudeste do país (Figura 1). Nessa época, o problema de deficiência de forragem, para os animais em pastejo, foi solucionado com uso de suplementação volumosa (292 kg ha-1 , em outubro de 2001, e 769 kg hä ${ }^{-1}$ em julho de 2002).

Durante o ano de 2001, a porcentagem de leguminosa na pastagem permaneceu constante durante os meses de janeiro a maio (26\% a $29 \%$ ), alcançou o maior valor (56\%) durante o final do período seco (outubro) e diminuiu com o início da época das chuvas (dezembro). Em 2002, a participação de $S$. guianensis na MS total de forragem seguiu o mesmo comportamento observado em 2001. Entretanto, o aumento da porcentagem de estilosantes durante a seca (julho) foi menos acentuado do que em 2002 (Figura 1). A variação na porcentagem de leguminosa ao longo do período experimental esteve condicionada, principalmente, à MS de $B$. decumbens nos diferentes meses do ano e, em julho de 2002, observou-se queda menor na MS de $B$. decumbens do que em outubro de 2001.

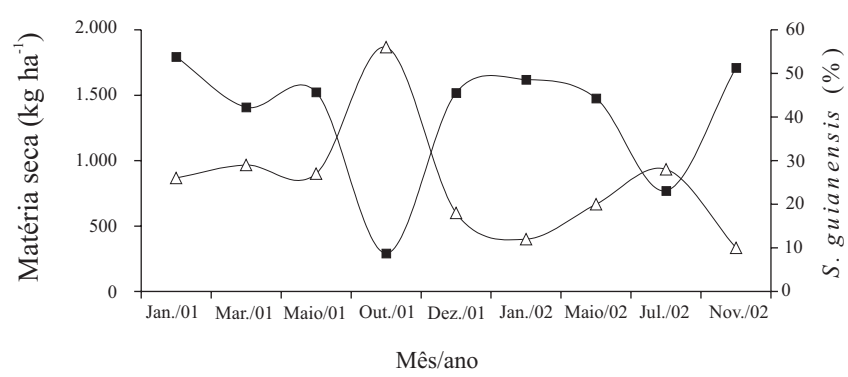

Figura 1. Rendimento de matéria seca de Brachiaria decumbens $(\square)$ e porcentagem de Stylosanthes guianensis $(\triangle)$, na pastagem, de acordo com o mês do ano. 
O decréscimo linear da MS de S. guianensis, ao longo do período experimental, resultou em menor valor médio de porcentagem de leguminosa na pastagem em 2002 (17,5\%), em relação a 2001 (31,2\%). Hou ve queda de $19,7 \mathrm{~kg} \mathrm{ha}^{-1}$ de MS por mês de avaliação (Figura 2). Nessa equação de regressão, X representa o mês de avaliação, em variação de 1 (janeiro de 2001) a 23 (novembro de 2002) e Y representa a MS de $S$. guianensis.

O decréscimo na porcentagem de leguminosa, na consorciação, pode ser atribuído à competição por água, luz e nutrientes, em geral, e entre plantas das diferentes espécies. B. decumbens, por ser uma planta de maior eficiência fotossintética (ciclo $\mathrm{C}_{4}$ ) em condições tropicais e de melhor adaptação às condições de solo de baixa fertilidade e topografia declivosa, foi mais competitiva do que a leguminosa (ciclo $\mathrm{C}_{3}$ ). Corroboram essa hipótese os dados de Paciullo et al. (2003), que encontraram taxas médias de acúmulo de MS, durante a primavera, de 4,0 kg ha dia-1 para a $S$. guianensis e de $25,0 \mathrm{~kg}$ ha dia ${ }^{-1}$ para a $B$. decumbens. A baixa persistência de leguminosas consorciadas com gramíneas é freqüentemente relatada na literatura. Seiffert \& Zimmer (1988) e Euclides et al. (1998) observaram reduções na porcentagem de leguminosas, quando consorciadas com B. decumbens de $30 \%$ para $6 \%$ e de $15 \%$ para $6 \%$, respectivamente, após três anos de pastejo.

Os dados de composição química da extrusa mostraram variação para todos os componentes, em relação aos períodos de avaliação, apesar dessa variação ter sido de pequena magnitude (Tabela 1). Não foi observada tendência consistente dos dados ao longo dos meses, mas destacaram-se os maiores teores de PB em

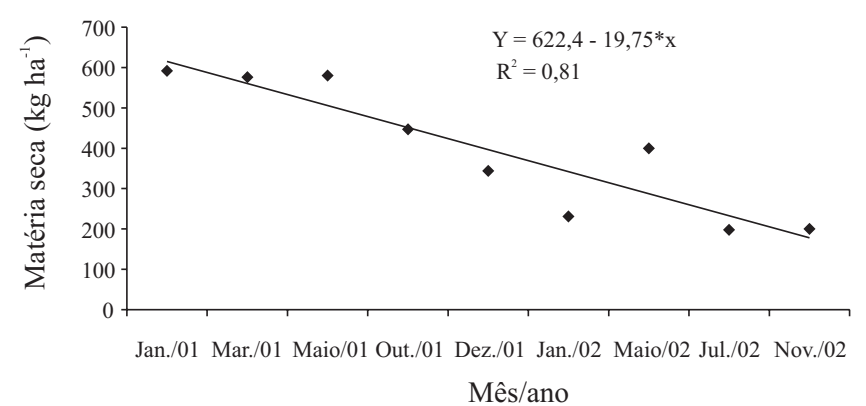

Figura 2. Rendimento de matéria seca de Stylosanthes guianensis, durante o período experimental. dezembro de 2001 e de FDN em janeiro de 2001 e 2002, e o menor teor de FDA em maio de 2001.

A DIVMS variou de 42,1\% a 48,0\%, dependendo do mês (Tabela 1). Estes dados são considerados baixos, quando comparados aos valores citados por Valle et al. (2001), de 50\% a 60\%. Os baixos valores encontrados são atribuídos aos elevados teores de FDN e FDA da extrusa.

O consumo total de MS foi máximo em maio de 2001 e não variou nos demais meses de avaliação (Tabela 2). O maior consumo de leguminosa foi também observado em maio de 2001, e o de gramínea, em novembro de 2002. Além de os menores teores de FDN e FDA do pasto, em maio de 2001, favorecerem o consumo, a maior participação da leguminosa na dieta, nesse mês, pode ter contribuído para a maior ingestão total de matéria seca. Os níveis de consumo estimados nesse estudo foram baixos, embora na literatura existam relatos de consumos de Brachiaria spp., com variação de 1,3\% a 2,6\% (Euclides et al., 2000). Os consumos de forragem ora relatados podem ter sido limitados pelos altos teores

Tabela 1. Teores de proteína bruta (PB), fibra em detergente neutro (FDN), fibra em detergente ácido (FDA) e digestibilidade in vitro da matéria seca (DIVMS) da extrusa, de acordo com o mês do ano(1).

\begin{tabular}{lcccc}
\hline Mês/ano & $\begin{array}{c}\text { PB } \\
\left(\mathrm{g} \mathrm{kg}^{-1}\right)\end{array}$ & $\begin{array}{c}\text { FDN } \\
\left(\mathrm{g} \mathrm{kg}^{-1}\right)\end{array}$ & $\begin{array}{c}\text { FDA } \\
\left(\mathrm{g} \mathrm{kg}^{-1}\right)\end{array}$ & $\begin{array}{c}\text { DIVMS } \\
(\%)\end{array}$ \\
\hline Janeiro/2001 & $77 \mathrm{~b}$ & $762 \mathrm{ab}$ & $439 \mathrm{a}$ & $42,1 \mathrm{~b}$ \\
Maio/2001 & $84 \mathrm{~b}$ & $702 \mathrm{c}$ & $353 \mathrm{~b}$ & $47,3 \mathrm{a}$ \\
Dezembro/2001 & $105 \mathrm{a}$ & $712 \mathrm{c}$ & $453 \mathrm{a}$ & $45,8 \mathrm{a}$ \\
Janeiro/2002 & $79 \mathrm{~b}$ & $794 \mathrm{a}$ & $465 \mathrm{a}$ & $46,5 \mathrm{a}$ \\
Maio/2002 & $86 \mathrm{~b}$ & $745 \mathrm{~b}$ & $427 \mathrm{a}$ & $46,4 \mathrm{a}$ \\
Novembro/2002 & $80 \mathrm{~b}$ & $739 \mathrm{~b}$ & $413 \mathrm{a}$ & $48,0 \mathrm{a}$ \\
\hline
\end{tabular}

${ }^{(1)}$ Médias seguidas pela mesma letra, na coluna, não diferem entre si pelo teste de Tukey a $5 \%$ de probabilidade.

Tabela 2. Consumo total de matéria seca e consumo diferenciado de gramínea e leguminosa, de acordo com o mês do ano ${ }^{(1)}$.

\begin{tabular}{lccc}
\hline Mês/ano & $\begin{array}{c}\text { Consumo total } \\
\text { (MS \% PV) }\end{array}$ & \multicolumn{2}{c}{ Consumo diferenciado (\% do total) } \\
\cline { 3 - 4 } & $1,50 \mathrm{~b}$ & $82,6 \mathrm{~b}$ & Gramínea \\
\hline Janeiro/2001 & $1,91 \mathrm{a}$ & $75,9 \mathrm{c}$ & $17,4 \mathrm{~b}$ \\
Maio/2001 & $1,56 \mathrm{~b}$ & $86,5 \mathrm{~b}$ & $24,1 \mathrm{a}$ \\
Dezembro/2001 & $1,59 \mathrm{~b}$ & $84,5 \mathrm{~b}$ & $13,5 \mathrm{~b}$ \\
Janeiro/2002 & $1,64 \mathrm{~b}$ & $83,1 \mathrm{~b}$ & $15,5 \mathrm{~b}$ \\
Maio/2002 & $1,57 \mathrm{~b}$ & $91,3 \mathrm{a}$ & $16,9 \mathrm{~b}$ \\
Novembro/2002 & & $8,7 \mathrm{c}$ \\
\hline
\end{tabular}

(1)Médias seguidas pela mesma letra, na coluna, não diferem entre si pelo teste de Tukey a 5\% de probabilidade. 
de FDN e baixos valores de DIVMS do pasto. Valores de FDN acima de 55\%-60\% correlacionam-se negativamente com o consumo de forragem (Mertens, 1987). Considerando-se que os teores de FDN observados foram sempre acima do valor crítico sugerido por Mertens (1987), admite-se que o consumo de forragem tenha sido limitado principalmente pelo enchimento ruminal, fenômeno normalmente observado para forrageiras tropicais.

A técnica da avaliação da proporção isotópica do ${ }^{13} \mathrm{C}$ nas fezes é usada para estimativa da proporção de espécies de ciclo fotossintético $\mathrm{C}_{3}$ e $\mathrm{C}_{4}$, presentes na dieta de bovinos. Não foi possível distinguir entre espécies de mesmo ciclo fotossintético. O consumo de leguminosa foi, em sua maior parte, constituído pelo $S$. guianensis, embora tenha sido observado, visualmente, em todos os períodos de avaliação, ramoneio das leguminosas arbóreas A. mangium e M. artemisiana pelos animais.

A quantidade de leguminosa é importante para condicionar sua ingestão pelos animais e influenciar o consumo total de forragem. Assim, os maiores consumos totais de MS foram observados quando as porcentagens de leguminosa nas dietas foram máximas (Figura 3). Lascano (1983) observou que a ingestão de forragem por novilhos, em pastagem consorciada de B. humidicola e Desmodium spp., durante o período seco do ano, foi maior (1,9\% do peso vivo) do que aquela verificada em pastagem exclusiva de gramínea (1,3\% do peso vivo). Euclides et al. (1998) evidencia-

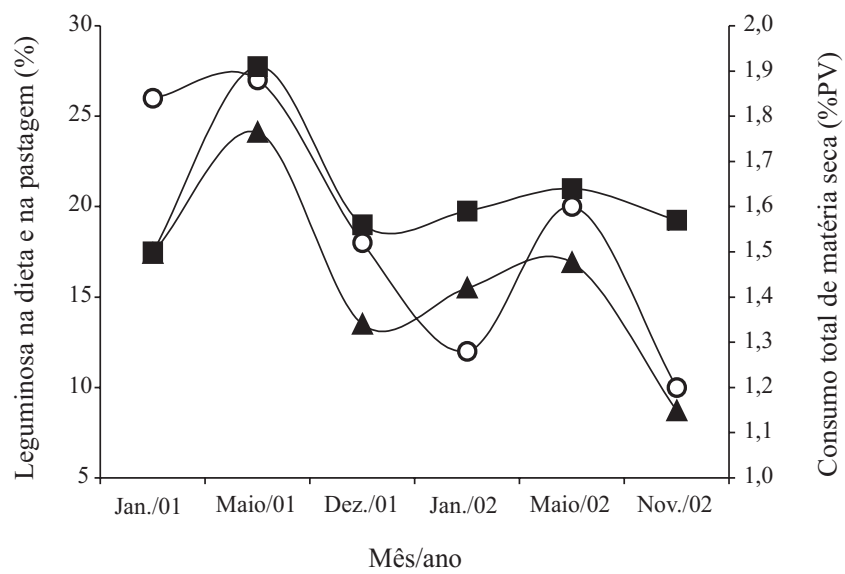

Figura 3. Porcentagens de leguminosa na pastagem $(\mathrm{O})$ e na dieta ( $\boldsymbol{\Delta}$ ), e consumo total ( $\boldsymbol{\square})$ de matéria seca (\% do peso vivo) de vacas mestiças, de acordo com o mês do ano. ram que a contribuição da leguminosa na dieta de animais resultou em melhor desempenho de bovinos em pastagem consorciada, relativamente àqueles mantidos em pastagem exclusiva de gramínea, mesmo quando a porcentagem de leguminosa na pastagem foi baixa.

\section{Conclusões}

1. A disponibilidade de forragem de $B$. decumbens é maior nos meses de novembro a maio, em relação ao meses de junho a outubro, enquanto a disponibilidade de S. guianensis decresce linearmente ao longo do tempo.

2.A composição químico-bromatológica e a digestibilidade in vitro da matéria seca da dieta selecionada por vacas em pastagem consorciada variam pouco e de forma inconsistente ao longo do ano.

3. S. guianensis participa da dieta de vacas mestiças ao longo de todo o ano e o maior consumo é observado no início da época seca.

4. Maior porcentagem de $S$. guianensis, na pastagem, contribui para aumentar o consumo de forragem.

\section{Referências}

BARCELOS, A.O.; VILELA, L.; MARTHA JÚNIOR, G.B. Utilização de banco de proteína como alternativa para a suplementação de vacas leiteiras. In: SIMPÓSIO SOBRE ALTERNATIVAS TECNOLÓGICAS, PROCESSUAIS E DE POLÍTICAS PÚBLICAS PARA PRODUÇÃO DE LEITE EM BASES SUSTENTÁVEIS, 5., 2003, Juiz de Fora. Anais. Juiz de Fora: Embrapa Gado de Leite, 2003. p.93-119.

BODDEY, R.M.; MACEDO, R.; TARRÉ, R.; FERREIRA, E.; OLIVEIRA, O.C.; RESENDE, C. de P.; CANTARUTTI, R.B.; PEREIRA, J.M.; ALVES, B.J.R.; URQUIAGA, S. Nitrogen cycling in Brachiaria pastures: the key to understanding the process of pasture decline. Agriculture, Ecosystems and Environment, v.103, p.389-403, 2004.

CANTARUTTI, R.B.; TARRÉ, R.M.; MACEDO, R.; CADISCH, G.; RESENDE, C.P.; PEREIRA, J.M.; BRAGA, J.M.; GOMEDE, J.A.; FERREIRA, E.; ALVES, B.J.R.; URQUIAGA, S.; BODDEY, R.M. The effect of grazing intensity and the presence of a forage legume on nitrogen dynamics in Brachiaria pastures in the Atlantic forest region of the South of Bahia, Brazil. Nutrient Cycling in Agroecosystem, v.64, p.257-271, 2002.

COSTA, N.L. Adubação nitrogenada e consorciação de capim-elefante (Pennisetum purpureum cv. Cameroon) com leguminosas forrageiras tropicais. Pesquisa Agropecuária Brasileira, v.30, p.401-408, 1995.

EUCLIDES, V.P.B.; MACEDO, M.C.M.; OLIVEIRA, M.P. Produção de bovinos em pastagens de Brachiaria spp. consorciadas com Calopogonium mucunoides nos Cerrados. Revista Brasileira de Zootecnia, v.27, p.238-245, 1998. 
EUCLIDES, V.P.B.; CARDOSO, E.G.; MACEDO, M.C.M.; OLIVEIRA, M.P. Consumo voluntário de Brachiaria decumbens cv. Basilisk e Brachiaria brizantha cv. Maradu sob pastejo. Revista Brasileira de Zootecnia, v.29, p.2200-2208, 2000. (Suplemento).

GILLER, K.; CADISCH, G. Future benefits from biological nitrogen fixation: an ecological approach to agriculture. Plant and Soil, v.174, p.255-277, 1995.

JINGURA, R.M.; SIBANDA, S.; HAMUDIKUWANDA, H. Yield and nutritive value of tropical forage legumes grown in semi-arid parts of Zimbabwe. Tropical Grassland, v.35, p.168-174, 2001.

LASCANO, C. Factores edáficos y climáticos que intervienen en el consumo y la selección de plantas forrajeras bajo pastoreo. In: PALADINES, O.; LASCANO, C. (Ed.). Germoplasma forrajero bajo pastoreo en pequeñas pascelas: metodologías de evaluación. Cali: Centro Internacional de Agricultura Tropical - CIAT, 1983. p.49-64.

LASCANO, C.E.; EUCLIDES, V.P.B. Nutritional quality and animal production of Brachiaria pastures. In: MILES, J.W.; MAASS, B.L.; VALLE, C.B. (Ed.). Brachiaria: biology, agronomy and improvement. Cali: CIAT; Campo Grande: Embrapa-CNPGC, 1996. p.106-123.

LUDLOW, M.M.; THOUGHTON, J.H.; JONES, R.J. A technique for determination of the proportion of $\mathrm{C}_{3}$ and $\mathrm{C}_{4}$ species in plant samples using stable natural isotopes of carbon. Journal of Agriculture Science, v.87, p.625-632, 1976.

MERTENS, D.R. Predicting intake and digestibility using mathematical models of ruminal function. Journal of Animal Science, v.64, p.1548-1558, 1987.

PACIULLO, D.S.C.; AROEIRA, L.J.M.; CARVALHO, C.A.B.; MORENZ, M.J.F. Taxa de acúmulo de forragem de Stylosanthes guianensis em pastagem consorciada. In: REUNIÃO ANUAL DA SOCIEDADE BRASILEIRA DE ZOOTECNIA, 40., 2003, Santa
Maria, RS. Anais. Santa Maria: Sociedade Brasileira de Zootecnia, 2003. 1 CD-ROM.

PEREIRA, J.M.; NASCIMENTO JUNIOR, D.; SANTANA, J.R.; CANTARUTTI, R.B.; LEÃO, M.I. Teor de proteína bruta e digestibilidade "in vitro" da matéria seca da forragem disponível e da dieta selecionada por bovinos em pastagem de B. humidicola (Rendle) Scweick, em monocultivo ou consorciado com leguminosas, submetida a diferentes taxas de lotação. Revista da Sociedade Brasileira de Zootecnia, v.21, p.104-117, 1992.

QUEIROZ, A.C.; SILVA, D.J. Análise de alimentos: métodos químicos e biológicos. Viçosa: Universidade Federal de Viçosa, 2002. 235p.

RODRIGUES, L.R.A.; RODRIGUES, T.J.D. Estabelecimento dos capins do gênero Cynodon em áreas de Brachiaria spp. In: WORKSHOP SOBRE O POTENCIAL FORRAGEIRO DO GÊNERO CYNODON, 1996, Juiz de Fora. Anais. Juiz de Fora: Embrapa Gado de Leite, 1996. p.8-21.

SEIFFERT, N.F.; ZIMMER, A.H. Contribución de Calopogonium mucunoides al contenido de nitrógeno en pasturas de Brachiaria decumbens. Pasturas Tropicales, v.10, p.8-13, 1988.

SOEST, P.J. van; ROBERTSON, J.B.; LEWIS, B. A method for dietary fiber, neutral detergent fiber, and non starch polysaccharides in relation to animal nutrition. Journal of Dairy Science, v.74, p.3583-3597, 1991.

TILLEY, J.M.A.; TERRY, R.A. A two stage technique for the in vitro digestion of forage crops. Journal of the British Grassland Society, v.18, p.104-111, 1963.

VALLE, C.B.; EUCLIDES, V.P.B.; MACEDO, M.C.M. Características das plantas forrageiras do gênero Brachiaria. In: SIMPÓSIO SOBRE MANEJO DE PASTAGEM, 17., 2001, Piracicaba. Anais. Piracicaba: Fealq, 2001. p.133-176.

Recebido em 2 de março de 2004 e aprovado em 6 de dezembro de 2004 